\title{
Top-quark condensate at finite temperature and electroweak symmetry restoration *
}

\author{
Bang-Rong Zhou \\ Department of Physics, Graduate School at Beijing, University of Science and Technology \\ of China, Academia Sinica, Beijing 100039, China \\ and \\ CCAST ( World Laboratory ) P.O.Box 8730, Beijing 100080, China
}

\begin{abstract}
The gap equation at finite temperature in the top-quark condensate scheme of electroweak symmetry breaking is proven to have the identical form in both the imaginary and the real time formalism of thermal field theory. By means of the gap equation, combined with the basic relation to define the vacuum expectation value $v$ of the effective Higgs field, we analyse the dependence on temperature $\mathrm{T}$ and chemical potential $\mu$ of the dynamical top-quark mass as the order parameter characteristic of symmetry breaking, and obtain the $\mu-T$ criticality curve for symmetry restoration. We find out that the critical temperature $T_{c}=2 v$ for $\mu=$ 0 and the critical chemical potential $\mu_{c}=2 \pi v / \sqrt{3}$ for $T=0$. When $\mu=0$, the top-quark mass near $T_{c}$ has the leading $\left(T_{c}^{2}-T^{2}\right)^{1 / 2}$ behavior with an extra factor dependent on temperature $T$ and the momentum cut-off $\Lambda$. However, it is generally argued that the symmetry restoration at $T \geq T_{c}$ is still a second-order phase transition.
\end{abstract}

PACS numbers: 12.15.-y, 11.15.Ex, 12.60.Fr, 12.90.+b

Key words: Gap equation, critical temperature and chemical potential, second-order phase tansition

\section{Introduction}

Research on electroweak symmetry phase transition at finite temperature is attracting much attentions [1]. It has not only fundamental field theory interest but also important practical implications to evolutiom of early universe and its cosmological results [2]. Many works have been made in the scheme where symmetry breaking is induced by elementary Higgs field [3-7]. On the other hand, it is also quite interesting to discuss phase transition of electroweak symmetry at high temperature in the scheme where symmetry breaking is realized dynamically, i.e. by the condensates of fermions. In view of that the top-quark condensate scheme $[8,9]$ is a good candicate of such dynamical scheme at zero-temperature, in this paper we will research its behavior at finite temperature.

*This project was suppored partially by National Natural Science Foundation of China and by Grant No.LWTZ-1298 of the Chinese Academy of Sciences. 
The top-quark condensate scheme is essentially a Nambu-Jona-Lasinio (NJL) model [10]. Without loss of essentiality of such model we will made our discussions only in the fermon bubble graph approximation and, to this order, completely neglect color gauge interactions. An obvious advantage of making this approximation is that the whole discussions could be conducted analytically. In this case, the only order parameter responsible for electroweak symmetry breaking is the dynamical top-quark mass $m(T, \mu)$ as the function of temperature $T$ and chemical potential $\mu$, which is proportional to the thermal top-quark condensate $\langle\bar{t} t\rangle_{T}$.

The paper is arranged as follows. In Sect. 2 we will derive the gap equation at finite temperature and finite chemical potential respectively from Feynman rules of the imaginary time formalism (ITF) and the real time formalism (RTF) of thermal field theory and show that both formalisms lead to the same result. In Sect. 3 an equation for critical chemical potential $\mu_{c}$ and critical temperature $T_{c}$ is derived and the $\mu_{c}-T_{c}$ curve and some numerical results of $\mu_{c}$ and $T_{c}$ are explicitly given. In Sect. 4 the temperature behavior of the dynamical quark mass $m(T, \mu)$ at $T \lesssim T_{c}$ is discussed and the phase transition's feature of the symmetry restoration is generally argued. Finally, in Sect. 5 we briefly come to our conclusions.

\section{Gap equation at finite temperature}

In the top-quark condensate scheme at zero temperature, one assumes that the neutral scalar sector of the four-fermion interactions with the coupling constant $G$

$$
\mathcal{L}_{4 F}^{N_{S}}=\frac{G}{4}(\bar{t} t)^{2}
$$

will lead to formation of the top-quark condensate $\langle\bar{t} t\rangle$ and generation of dynamical topquark mass $m(0)$. In the bubble graph approximation, the gap equation is [9]

$$
m(0)=-\frac{G}{2}\langle\bar{t}(x) t(x)\rangle
$$

Equation (2.2) leads to

$$
m(0)=\frac{G}{2} d_{t}(R) \int \frac{d^{4} k}{(2 \pi)^{4}} \operatorname{tr}\left[\frac{i}{\not k-m(0)+i \varepsilon}\right]
$$

and furthermore,

$$
1=\frac{G \Lambda^{2} d_{t}(R)}{8 \pi^{2}}\left[1-\frac{m^{2}(0)}{\Lambda^{2}} \ln \left(\frac{\Lambda^{2}}{m^{2}(0)}+1\right)\right]
$$

where $\Lambda$ is the momentum cut-off in 4-dimension Euclidean space and $d_{t}(R)$ is the dimension of the $S U_{c}(3)$ representation $R$ of the top-quark.

The extension of Eq. (2.2) to finite temperature $T$ and finite chemical potential $\mu$ will be

$$
m(T, \mu)=-\frac{G}{2}\langle\bar{t}(x) t(x)\rangle_{T}
$$

where $\langle\bar{t}(x) t(x)\rangle_{T}$ represents thermal expectation value. However, since thermal field theory has two kinds of formalism: ITF and RTF [11], we must show that the extended gap equations (2.5) are equivalent in the two formalisms. In the following we will prove that they are in fact identical.

1) ITF: In this formalism the Feynman rules at zero-temperature should be modified 
according to that

fermion propagator:

$$
\begin{gathered}
\frac{i}{\not k-m(0)+i \varepsilon} \rightarrow \frac{\not k+m}{\left(\omega_{n}+i \mu\right)^{2}+\mathbf{k}^{2}+m^{2}} \\
k^{0}=-i \omega_{n}+\mu, \quad \omega_{n}=\frac{(2 n+1) \pi}{\beta}, \quad n=0, \pm 1, \pm 2, \ldots
\end{gathered}
$$

with the denotations $\beta=1 / T$ and $m \equiv m(T, \mu)$;

the momentum integral of a fermion loop:

$$
\int \frac{d^{4} k}{(2 \pi)^{4}} \rightarrow \int \frac{d^{3} k}{(2 \pi)^{3}} T \sum_{n=-\infty}^{\infty}
$$

As a result, equation (2.5) can be changed into

$$
1=2 G d_{t}(R) \int \frac{d^{3} k}{(2 \pi)^{3}} T \sum_{n=-\infty}^{\infty} \frac{1}{\left(\omega_{n}+i \mu\right)^{2}+\omega_{k}^{2}}, \quad \omega_{k}^{2} \equiv \omega_{k}^{2}(T, \mu)=\mathbf{k}^{2}+m^{2}
$$

The sum in Eq. (2.8) can be done by means of the integral formula

$$
T \sum_{n=-\infty}^{\infty} f[z=(2 n+1) \pi / \beta+i \mu]=\frac{i}{4 \pi} \oint_{C} d z f(z) \tan \left[\frac{1}{2} \beta(z-i \mu)\right]
$$

where the contour $C$ contains the straight lines parallel to the real axis which extend from point $(i \mu-\infty)$ to $(i \mu+\infty)$ then goes back to the point $(i \mu-\infty)$ but round all the points $i \mu+(2 n+1) \pi / \beta(n=0, \pm 1, \pm 2, \ldots)$. By appropriately modifying the contour $C$, we will have that

$$
\begin{aligned}
& T \sum_{n=-\infty}^{\infty} f[z=(2 n+1) \pi / \beta+i \mu]= \\
& \quad \frac{1}{2 \pi} \int_{-\infty}^{\infty} d z f(z)-\frac{1}{2 \pi} \int_{i(\mu-\varepsilon)-\infty}^{i(\mu-\varepsilon)+\infty} d z f(z)\{\exp [i \beta(z-i \mu)]+1\}^{-1}+ \\
& \quad \frac{1}{2 \pi} \int_{i(\mu+\varepsilon)+\infty}^{i(\mu+\varepsilon)-\infty} d z f(z)\{\exp [-i \beta(z-i \mu)]+1\}^{-1}
\end{aligned}
$$

where

$$
f(z)=1 /\left(z^{2}+\omega_{k}^{2}\right)
$$

Substituting Eq. (2.10) into Eq. (2.8), we obtain the gap equation at finite temperature

$$
\begin{aligned}
1= & 2 G d_{t}(R)\left\{\int \frac{d^{3} k d \overline{k^{0}}}{(2 \pi)^{4}} \frac{1}{\bar{k}^{2}+\omega_{k}^{2}}\right. \\
& \left.-\int \frac{d^{3} k}{(2 \pi)^{3} 2 \omega_{k}}\left\{\left\{\exp \left[\beta\left(\omega_{k}-\mu\right)\right]+1\right\}^{-1}+\left\{\exp \left[\beta\left(\omega_{k}+\mu\right)\right]+1\right\}^{-1}\right\}\right\}
\end{aligned}
$$


Then by means of the Euclidean 4-dimension momentum cut-off $\Lambda$ Eq. (2.12) will be reduced to

$$
\begin{aligned}
1= & \frac{G \Lambda^{2} d_{t}(R)}{8 \pi^{2}}\left\{1-\frac{m^{2}}{\Lambda^{2}} \ln \left(\frac{\Lambda^{2}}{m^{2}}+1\right)\right. \\
& \left.-\frac{4}{\Lambda^{2}} \int_{0}^{\infty} d|\mathbf{k}| \frac{|\mathbf{k}|^{2}}{\omega_{k}}\left\{\left\{\exp \left[\beta\left(\omega_{k}-\mu\right)\right]+1\right\}^{-1}+\left\{\exp \left[\beta\left(\omega_{k}+\mu\right)\right]+1\right\}^{-1}\right\}\right\}
\end{aligned}
$$

The 3-dimension momentum integral in the second term of right-handed side of Eq. (2.12) is convergent and this allows us to have extended the upper limit of the integral to infinite. Such an extension with the advantage of greatly simplifying the calculations seems to have a little inconsistence with the fact that the four-fermion Lagrangian (2.1) only express an effective interactions defined at the scales below the compositeness scale $\Lambda$. However, since the 3 -dimension momentum integral from $\Lambda$ to infinity only contributes a very little part of the total one, the extension can be viewed as a good approximation and will not change the essential results. When $\mu=0$ and $\beta \rightarrow \infty(T \rightarrow 0), m \equiv m(T, \mu)$ will become $m(0)$ and Eq. (2.13) will go back to the gap equation (2.4) in zero-temperature case.

2) RTF: In this formalism, all fields and interactions will be doubled. The four-fermion interactions (2.1) should be replaced by

$$
\overline{\mathcal{L}}_{4 F}^{N_{S}}=\frac{G}{4} \sum_{r=1,2}(-1)^{r+1}\left(\bar{t}^{(r)} t^{(r)}\right)^{2}
$$

where $r=1$ means physical fields and $r=2$ ghost fields. The physical fields and the ghost fields interact only through the propagator. Thus the thermal propagator of the fermion will be a $2 \times 2$ matrix:

$$
i \mathbf{S}(k, m)=\mathbf{M}\left(\begin{array}{cc}
S(k, m) & 0 \\
0 & S^{*}(k, m)
\end{array}\right) \mathbf{M}
$$

where $2 \times 2$ matrix

$$
\mathbf{M}=\left(\begin{array}{cc}
\cos \theta\left(k^{0}, \mu\right) & -\exp \left[-\beta\left(k^{0}-\mu\right) / 2\right] \sin \theta\left(k^{0}, \mu\right) \\
\exp \left[-\beta\left(k^{0}-\mu\right) / 2\right] \sin \theta\left(k^{0}, \mu\right) & \cos \theta\left(k^{0}, \mu\right)
\end{array}\right)
$$

with

$$
\sin \theta\left(k^{0}, \mu\right)=\frac{\theta\left(k^{0}\right) \exp \left[-\beta\left(k^{0}-\mu\right) / 4\right]-\theta\left(-k^{0}\right) \exp \left[\beta\left(k^{0}-\mu\right) / 4\right]}{\left\{\exp \left[\beta\left(k^{0}-\mu\right) / 2\right]+\exp \left[-\beta\left(k^{0}-\mu\right) / 2\right]\right\}^{1 / 2}}
$$

and

$$
S(k, m)=\frac{i}{\not k-m+i \varepsilon}
$$

Because the fermion mass $m$ is generated merely dynamically through the thermal topquark condensate $\langle\bar{t}(x) t(x)\rangle_{T}$, the thermal fermon propagator $i S^{a b}(k, m)$ will submit to the following equations

$$
i S^{a b}(k, m)=i S^{a b}(k, 0)+i S^{a c}(k, 0) i(-1)^{c} \Sigma^{c}\left[i S^{c b}(k, m)\right] \quad a, b, c=1,2
$$

where repeat of an index means its sum and $i(-1)^{c} \Sigma^{c}$ represents the contribution of the fermion loop connected to a $c$-type four-fermion vertex with

$$
\Sigma^{c}=\frac{G}{2} d_{t}(R) \int \frac{d^{4} k}{(2 \pi)^{4}} \operatorname{tr}\left[i S^{c c}(k, m)\right] \quad(\text { no sum of } c)
$$


By means of the matrix propagator (2.15), Eq. (2.19) can be explicitly reduced to

$$
\begin{aligned}
& \left(\begin{array}{cc}
S(k, m) & 0 \\
0 & S^{*}(k, m)
\end{array}\right)=\left(\begin{array}{cc}
S(k, 0) & 0 \\
0 & S^{*}(k, 0)
\end{array}\right) \\
& \quad+\left(\begin{array}{cc}
S(k, 0) & 0 \\
0 & S^{*}(k, 0)
\end{array}\right) \mathbf{M} i\left(\begin{array}{cc}
\Sigma^{1} & 0 \\
0 & \Sigma^{2}
\end{array}\right) \mathbf{M}\left(\begin{array}{cc}
S(k, m) & 0 \\
0 & S^{*}(k, m)
\end{array}\right)
\end{aligned}
$$

The formula (2.20) gives that

$$
\begin{gathered}
\Sigma^{1}=m G 2 d_{t}(R) \int \frac{d^{4} k}{(2 \pi)^{4}}\left[\frac{i}{k^{2}-m^{2}+i \varepsilon}-2 \pi \delta\left(k^{2}-m^{2}\right) \sin ^{2} \theta\left(k^{0}, \mu\right)\right] \\
\Sigma^{2}=\Sigma^{1^{*}}
\end{gathered}
$$

After counter clockwise and clockwise wick rotation of the integral contour of $k^{0}$ respectively, it is easy to verify that $\Sigma^{1}$ and $\Sigma^{2}$ are both real and then from Eqs (2.23) and (2.16) we may write the factor in Eq. (2.21)

$$
\mathbf{M} i\left(\begin{array}{cc}
\Sigma^{1} & 0 \\
0 & \Sigma^{2}
\end{array}\right) \mathbf{M}=-i \Sigma^{1}\left(\begin{array}{cc}
1 & 0 \\
0 & -1
\end{array}\right)
$$

Substituting Eq. (2.24) into Eq. (2.21), we find out

$$
S(k, m)=\frac{i}{\not k-\Sigma^{1}+i \varepsilon}, \quad S^{*}(k, m)=\frac{-i}{\not k-\Sigma^{1}-i \varepsilon}
$$

Hence, we may identify $\Sigma^{1}$ with the dynamical fermion mass $m \equiv m(T, \mu)$ and then from Eq. (2.22) obtain

$$
1=G 2 d_{t}(R) \int \frac{d^{4} k}{(2 \pi)^{4}}\left[\frac{i}{k^{2}-m^{2}+i \varepsilon}-2 \pi \delta\left(k^{2}-m^{2}\right) \sin ^{2} \theta\left(k^{0}, \mu\right)\right]
$$

which is the gap equation in RTF. In fact, it is easy to see that Eq. (2.26) can be obtained from Eq. (2.3) simply through the replacement of the fermion propagator

$$
\frac{i}{\not k-m(0)+i \varepsilon} \rightarrow i S^{11}(k, m)=\frac{i}{\not k-m+i \varepsilon}-2 \pi \delta\left(k^{2}-m^{2}\right)(\not k+m) \sin ^{2} \theta\left(k^{0}, \mu\right)
$$

includung the replacement $m(0) \rightarrow m$. The replacement $(2.27)$ is also valid in some other simple problems. However, only through the above derivation based on the matrix propagator in RTF it can be believed that correctness of the replacement (2.27) has been proven rigorously.

Equation (2.26) can be changed into the form of Eq. (2.13) after the Wick rotation, angular integration and introduction of the Euclidean 4-dimension momentum cut-off $\Lambda$. This indicates that the gap equation (2.5) are identical in ITF and RTF. Furthermore, we can eliminate the four-fermion coupling constant $G$ in Eq. (2.13) by means of the zero-temperature gap equation (2.4) and transform Eq. (2.13) into

$$
\begin{aligned}
& m^{2}(0) \ln \left(\frac{\Lambda^{2}}{m^{2}(0)}+1\right)=m^{2} \ln \left(\frac{\Lambda^{2}}{m^{2}}+1\right) \\
& \quad+4 \int_{0}^{\infty} d|\mathbf{k}| \frac{|\mathbf{k}|^{2}}{\omega_{k}}\left\{\left\{\exp \left[\beta\left(\omega_{k}-\mu\right)\right]+1\right\}^{-1}+\left\{\exp \left[\beta\left(\omega_{k}+\mu\right)\right]+1\right\}^{-1}\right\}
\end{aligned}
$$

which will be the starting equation in our following discussions. 


\section{The $\mu-T$ criticality equation and curve}

For the zero-temperature top-quark condensate scheme of electroweak symmetry breaking in the fermion bubble graph approximation, we have the following basic relation [9]

$$
\frac{4 \sqrt{2} \pi^{2}}{3 G_{F}}=\frac{8 \pi^{2}}{3} v^{2}=m^{2}(0) \ln \left[\frac{\Lambda^{2}}{m^{2}(0)}+1\right]
$$

where $G_{F}$ is the Fermi constant and $v$ is the vacuum expectation value of the Higgs field in the standard electroweak model. As a result of Eq. (3.1), equation (2.28) may be reduced to

$$
\begin{aligned}
\frac{8 \pi^{2}}{3} v^{2}= & m^{2} \ln \left(\frac{\Lambda^{2}}{m^{2}}+1\right) \\
& +4 \int_{0}^{\infty} d|\mathbf{k}| \frac{|\mathbf{k}|^{2}}{\omega_{k}}\left\{\left\{\exp \left[\beta\left(\omega_{k}-\mu\right)\right]+1\right\}^{-1}+\left\{\exp \left[\beta\left(\omega_{k}+\mu\right)\right]+1\right\}^{-1}\right\}
\end{aligned}
$$

We notice that the four-fermion coupling constant $G$ and the zero-temperature dynamical fermion mass $m(0)$ have disappeared from Eq. (3.2) and they have been replaced by the vacuum expectation value $v$ of the Higgs field.

It is seen from Eq. (3.2) that for a fixed chemical potential $\mu$, the second term in the right-handed side of the equation will be growing as the temperature $T$ is going up $(\beta$ is going down). However, in order to keep the left-handed side of the equation to be a constant, the first term in the right-handed side must be descending and eventually, one will arrive at a critical temperature $T_{c}$, where $m\left(T_{c}, \mu\right)=0$, i.e. $\langle\bar{t} t\rangle_{T_{c}}=0$. This means that electroweak symmetry will be restored at the temperature $T \geq T_{c}$ when $\mu$ is fixed. The similar situation will happen for a fixed temperature $T$. That is, we will have a critical chemical potential $\mu_{c}$, and $m\left(T, \mu_{c}\right)=0$ for $\mu \geq \mu_{c}$ when $T$ is fixed. The equation which determines $\mu_{c}$ and $T_{c}$ can be obtained by setting $m=0$ in Eq. (3.2). The result is

$$
\frac{2 \pi^{2}}{3} v^{2}=T^{2} \int_{0}^{\infty} d x\left[\frac{x}{\exp (x-r)+1}+\frac{x}{\exp (x+r)+1}\right]
$$

where the denotations $x=\beta|\mathbf{k}|$ and $r=\beta \mu$ have been used. By means of the formula $[12]$

$$
\int_{0}^{\infty} d x \frac{x^{s}}{\exp (x \mp r)+1}=\exp ( \pm r) \Gamma(s+1) \Phi[-\exp ( \pm r), s+1,1]
$$

with the definition of Lerch's transcendent function

$$
\Phi(z, s, a)=\sum_{k=0}^{\infty} \frac{z^{k}}{(k+a)^{s}}
$$

equation (3.3) becomes

$$
\frac{2 \pi^{2}}{3} v^{2}=T^{2}\{\exp (\mu / T) \Phi[-\exp (\mu / T), 2,1]+\exp (-\mu / T) \Phi[-\exp (-\mu / T), 2,1]\}
$$

In view of that when $\mu=0, \Phi[-\exp (-\mu / T), 2,1]=\Phi[-1,2,1]=\pi^{2} / 12$, Eq. (3.6) will lead to

$$
T_{c}=2 v, \quad \text { when } \mu=0
$$


On the other hand, when $T \rightarrow 0$, i.e. $r=\mu / T \rightarrow \infty$ if $\mu$ is fixed, we can rewrite the factor $T^{2}=\mu^{2} / r^{2}$ in Eq. (3.3) and then, in terms of the limits

$$
\lim _{r \rightarrow \infty} \frac{1}{r^{2}} \int_{0}^{\infty} d x \frac{x}{\exp (x \mp r)+1}=\left\{\begin{array}{c}
\frac{1}{2} \\
0
\end{array}\right.
$$

obtain from Eq. (3.3) that

$$
\mu_{c}=\frac{2 \pi}{\sqrt{3}} v, \quad \text { when } T=0
$$

From Eq. (3.6), we may draw the general $\mu-T$ criticality curve which is displayed in Figure 1.

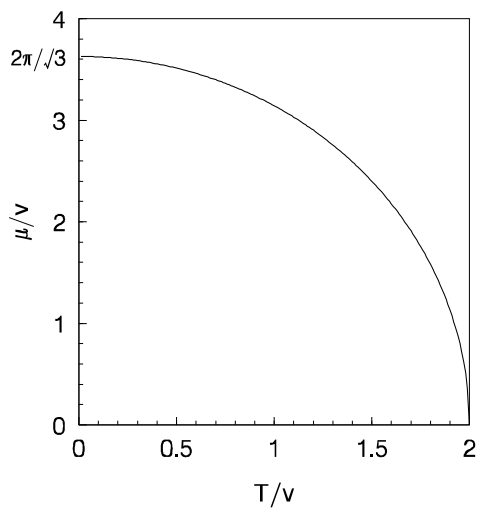

Fig. 1 The critical chemical potential $\mu_{c}$-the critical temperature $T_{c}$ curve (scaled in the Higgs vacuum expectation value $v$ ).

In order to show numerical estimations of $\mu_{c}$ and $T_{c}$, we take $v=246 \mathrm{GeV}$ and list the values of some special points in the $\mu_{c}-T_{c}$ curve in Table 1.

Table 1. Some special values of $\mu_{c}$ and $T_{c}$ when the Higgs vacuum expectation value $v=246 \mathrm{GeV}$ is taken.

\begin{tabular}{lllllllllll}
\hline$T_{c}(\mathrm{GeV})$ & 0 & 10 & 50 & 100 & 300 & 400 & 450 & 485 & 490 & 492 \\
\hline & & & & & & & & & & \\
$\mu_{c}(\mathrm{GeV})$ & 892.4 & 892.2 & 888 & 874 & 707 & 520 & 361 & 150 & 80 & 0 \\
\hline
\end{tabular}

The expression (3.7) gives the highest critical temperature $T_{c}=2 v=492 \mathrm{GeV}$ which appears when $\mu=0$. Such value of $T_{c}$ is higher than the temperature of electroweak symmetry restoration $T_{c}=\sqrt{2} v=347 \mathrm{GeV}$ obtained in the theory with elementary Higgs scalar field if electroweak gauge interactions are taken off ( and inclusion of these interactions will only make $T_{c}$ become lower.) [7]. This indicates a difference between dynamical 
and non-dynamical scheme of electroweak symmetry breaking. The formula (3.9) also shows that even if at $T=0$ the symmetry restoration could still occur if the chemical potential $\mu \geq 2 \pi v / \sqrt{3}=892 \mathrm{GeV}$, at least that is the case theoretically. Generally, $T_{c}$ always monotonically decreases as $\mu$ increases.

\section{Critical behavior of dynamical fermion mass}

The critical behavior of the dynamical fermion mass $m \equiv m(T, \mu)$ at $T \lesssim T_{c}$ can be obtained from Eq. (3.2) by high temperature expansion of the integrals in the equation. For this purpose, we rewrite Eq. (3.2) as follows:

$$
\frac{8 \pi^{2}}{3} v^{2}=m^{2} \ln \left(\frac{\Lambda^{2}}{m^{2}}+1\right)+8 T^{2}\left[I_{3}(y,-r)+I_{3}(y, r)\right]
$$

where we have generally defined

$$
I_{n}(y, \mp r)=\frac{1}{\Gamma(n)} \int_{0}^{\infty} d x \frac{x^{n-1}}{\left(x^{2}+y^{2}\right)^{1 / 2}}\left\{\exp \left[\left(x^{2}+y^{2}\right)^{1 / 2} \mp r\right]+1\right\}^{-1}
$$

with the Gamma function $\Gamma(n)$ and the denotation $y=\beta m$. By means of the recursion relations

$$
\frac{d I_{n+1}(y, \mp r)}{d y}=-\frac{y}{n} I_{n-1}(y, \mp r)
$$

for $n>1$ and Eq.(3.4) we obtain

$$
\begin{aligned}
I_{3}(y,-r)+I_{3}(y, r) & =-\frac{1}{2} \int_{0}^{y} d x x\left[I_{1}(x,-r)+I_{1}(x, r)\right] \\
& +\frac{1}{2}\{\exp (r) \Phi[-\exp (r), 2,1]+\exp (-r) \Phi[-\exp (-r), 2,1]\}
\end{aligned}
$$

On purpose to calculate $\left[I_{1}(x,-r)+I_{1}(x, r)\right]$, we make use of the formula

$$
\frac{1}{e^{\theta}+1}=\frac{1}{2}-2 \sum_{l=0}^{\infty} \frac{\theta}{(2 l+1)^{2} \pi^{2}+\theta^{2}}
$$

and write that

$$
\begin{aligned}
& I_{1}(y,-r)+I_{1}(y, r)=\int_{0}^{\infty} \frac{d x}{\left(x^{2}+y^{2}\right)^{1 / 2}} \\
& \quad-4 \sum_{l=0}^{\infty} \int_{0}^{\infty} \frac{d x}{x^{2}+(2 l+1)^{2} \pi^{2}+y^{2}-r^{2}}\left\{1+\frac{4 r^{2}(2 l+1)^{2} \pi^{2}}{\left[x^{2}+(2 l+1)^{2} \pi^{2}+y^{2}-r^{2}\right]^{2}}\right\}^{-1}
\end{aligned}
$$

Assume that

$$
\xi=\frac{r^{2}}{x^{2}+(2 l+1)^{2} \pi^{2}+y^{2}} \leq \frac{r^{2}}{\pi^{2}}<1
$$

and

$$
\eta=\frac{y^{2}}{x^{2}+(2 l+1)^{2} \pi^{2}} \leq \frac{y^{2}}{\pi^{2}}<1
$$


then we can make the power series expansion of the integrand in the second term of the right-handed side of Eq. (4.6) first in $\xi$ then in $\eta$. Retaining the terms up to the order $r^{\alpha} y^{\beta}$ with $\alpha+\beta \leq 6$ we will be able to write

$$
I_{1}(y,-r)+I_{1}(y, r)=\lim _{\varepsilon \rightarrow 0}\left(I_{1 a}^{\varepsilon}+I_{1 b}^{\varepsilon}\right)+\sum_{i=2,4,6} f_{i} y^{i}+\sum_{i=2,4,6} g_{i}\left(y^{2}\right) r^{i}
$$

where

$$
I_{1 a}^{\varepsilon}=\int_{0}^{\infty} d x \frac{x^{-\varepsilon}}{\left(x^{2}+y^{2}\right)^{1 / 2}} \text { and } I_{1 b}^{\varepsilon}=-4 \sum_{l=0}^{\infty} \int_{0}^{\infty} d x \frac{x^{-\varepsilon}}{x^{2}+(2 l+1)^{2} \pi^{2}}
$$

are two divergent integrals which have been regularized by the factor $x^{-\varepsilon}(0<\varepsilon \ll 1)$ added in the integrands [5], $f_{i}(i=2,4,6)$ are finite constants and $g_{i}\left(y^{2}\right)(i=2,4,6)$ are the functions of $y^{2}$. By means of the formula connected to the Euler beta function $B(p, q)$ $[12]$

$$
\int_{0}^{\infty} d x \frac{x^{m-1}}{\left(1+b x^{a}\right)^{m+n}}=a^{-1} b^{-m / a} B(m / a, m+n-m / a) \quad(a, b>0)
$$

it follows that

$$
\begin{aligned}
I_{1 a}^{\varepsilon} & =\frac{1}{2} y^{-\varepsilon} B\left(\frac{1-\varepsilon}{2}, \frac{\varepsilon}{2}\right)=\frac{1}{2} y^{-\varepsilon} \Gamma\left(\frac{1-\varepsilon}{2}\right) \Gamma\left(\frac{\varepsilon}{2}\right) / \Gamma\left(\frac{1}{2}\right) \\
& =\frac{1}{\varepsilon}-\ln \frac{y}{2}+O(\varepsilon)
\end{aligned}
$$

and in terms of the generalized Riemann zeta function $\zeta(s, a)$ and the Riemann zeta function $\zeta(s)[12]$ we obtain

$$
\begin{aligned}
I_{1 b}^{\varepsilon} & =-\frac{1}{(2 \pi)^{\varepsilon}} \zeta\left(1+\varepsilon, \frac{1}{2}\right)=-\frac{1}{(2 \pi)^{\varepsilon}}\left(2^{1+\varepsilon}-1\right) \zeta(1+\varepsilon) \\
& =-\frac{1}{\varepsilon}-\gamma+\ln \frac{\pi}{2}+O(\varepsilon)
\end{aligned}
$$

where $\gamma$ is the Euler constant. As a result, the sum in Eq. (4.9)

$$
\lim _{\varepsilon \rightarrow 0}\left(I_{1 a}^{\varepsilon}+I_{1 b}^{\varepsilon}\right)=-\ln \frac{y}{\pi}-\gamma
$$

eventually become finite. The other terms in Eq. (4.9) are all finite and can be expressed by the Riemann zeta functions $\zeta(s)(s=3,5,7)$. Based on the resulting expression of $I_{1}(y,-r)+I_{1}(y, r)$ and the result from Eq. (4.4), the equation (4.1) finally becomes

$$
\begin{aligned}
& \frac{8 \pi^{2}}{3} v^{2}=m^{2} \ln \left(\frac{\Lambda^{2}}{m^{2}}+1\right)+4 T^{2}\{\exp (r) \Phi[-\exp (r), 2,1]+\exp (-r) \Phi[-\exp (-r), 2,1]\} \\
& +2 m^{2}\left\{\ln \frac{y}{\pi}-\frac{1}{2}+\gamma-\frac{7}{4} \zeta(3)\left[(y / 2 \pi)^{2}+4(r / 2 \pi)^{2}\right]\right. \\
& \quad+\frac{31}{8} \zeta(5)\left[(y / 2 \pi)^{4}+12(y / 2 \pi)^{2}(r / 2 \pi)^{2}+8(r / 2 \pi)^{4}\right] \\
& \left.\quad-\frac{127}{16} \zeta(7)\left[\frac{5}{4}(y / 2 \pi)^{6}+30(y / 2 \pi)^{4}(r / 2 \pi)^{2}+60(y / 2 \pi)^{2}(r / 2 \pi)^{4}+16(r / 2 \pi)^{6}\right]\right\}
\end{aligned}
$$


which is valid in the conditions (4.7) and (4.8). We indicate that , at the temperature $T \lesssim T_{c}$, the condition (4.7) is easily satisfied since in this case we will have $m \approx 0$ thus $y / \pi=m / T \pi \ll 1$. However, the condition (4.8) can not always be satisfied at a definite $T_{c}$. This is because, by observation of the $\mu-T$ criticality curve in Figure 1, for sufficient low $T_{c}$ we will have $\mu_{c} / T_{c} \pi \geq 1$. Therefore, the condition (4.8) means that Eq. (4.15) is applicable only to the case of high $T_{c}$ where $\mu_{c} / T_{c} \pi<1$ is satisfied, i.e. when $\mu \neq 0$, Eq. (4.15) can only be considered as the high temperature expansion of Eq. (4.1).

The temperature dependence of $m \equiv m(T, \mu)$ at $T \lesssim T_{c}$ can be derived from Eq. (4.15). Since $m \approx 0$ when $T \lesssim T_{c}$, we will be able to omit the terms of the order $(m / T)^{4}\left(y^{4}\right)$ and above in Eq. (4.15). In addition, if $\mu / T \pi<1$ is assumed and $\Lambda^{2} / m^{2} \gg 1$ is considered, then Eq. (4.15) will be reduced to

$$
\begin{aligned}
\frac{8 \pi^{2}}{3} v^{2}= & 4 T^{2}\{\exp (r) \Phi[-\exp (r), 2,1]+\exp (-r) \Phi[-\exp (-r), 2,1]\} \\
& +2 m^{2}\left[\ln (\Lambda / T \pi)-\frac{1}{2}+\gamma-7 \zeta(3)(r / 2 \pi)^{2}+31 \zeta(5)(r / 2 \pi)^{4}-127 \zeta(7)(r / 2 \pi)^{6}\right]
\end{aligned}
$$

Considering the equation (3.6) which determines the critical temperature $T_{c}$ (in which $T$ should be replaced by $T_{c}$ ) we may change Eq. (4.16) into

$$
\begin{aligned}
m^{2}= & 2\left[T_{c}^{2}\left\{\exp \left(r_{c}\right) \Phi\left[-\exp \left(r_{c}\right), 2,1\right]+\exp \left(-r_{c}\right) \Phi\left[-\exp \left(-r_{c}\right), 2,1\right]\right\}\right. \\
& \left.-T^{2}\{\exp (r) \Phi[-\exp (r), 2,1]+\exp (-r) \Phi[-\exp (-r), 2,1]\}\right] / \\
& {\left[\ln (\Lambda / T \pi)-\frac{1}{2}+\gamma-7 \zeta(3)(r / 2 \pi)^{2}+31 \zeta(5)(r / 2 \pi)^{4}-127 \zeta(7)(r / 2 \pi)^{6}\right] }
\end{aligned}
$$

where the denotation $r_{c}=\mu / T_{c}$ has been used. The equation (4.17) gives the temperature dependence of $m$ at $T \lesssim T_{c}$ when $\mu<T \pi$. For the special case with $\mu=0$, it can be reduced to

$$
m=b(T)\left(T_{c}^{2}-T^{2}\right)^{1 / 2}, \quad b(T)=\pi / \sqrt{3}\left(\ln \frac{\Lambda}{T \pi}-\frac{1}{2}+\gamma\right)^{1 / 2}
$$

By Eq. (2.5), the dynamical mass $m$ is proportional to the fermion condensate $\langle\bar{t} t\rangle_{T}$ at temperature $T$ and can be regarded as the order parameter responsible for electroweak symmetry breaking. Therefore, the order parameter $m$ has the leading temperature behavior $\left(T_{c}^{2}-T^{2}\right)^{1 / 2}$ at $T \lesssim T_{c}$, which is the same as the temperature behavior of the order parameter in the theory with elementary Higgs scalar field [7], but $m$ now contains an additional factor dependent on $\ln (\Lambda / T \pi)$. For the case with non-zero chemical potential $\mu \neq 0$, the temperature behavior of $m$ at $T \lesssim T_{c}$ should be determined by Eq. (4.17).

Now let us return the case with $\mu=0$. It may be generally shown that the temperature behavior $\left(T_{c}^{2}-T^{2}\right)^{1 / 2}$ of the order parameter $m$ indicates that the symmetry restoration at $T \geq T_{c}$ will be a second-order phase transition in spite of the extra factor containing $\ln (\Lambda / \bar{T} \pi)$ in $m$. This conclusion is the same as the one reached in the model with elementary Higgs fields [7]. In fact, for a given model of fermion condensate, the momentum cut-off or compositeness scale $\Lambda$ is a fixed finite constant. In addition, since $T_{c} \neq 0$, the extra factor containing $\ln (\Lambda / T \pi)$ in $m$ will have no singularity at $T=T_{c}$. Consequently, the feature of the phase transition will be determined completely by the temperature behavior $\left(T_{c}^{2}-T^{2}\right)^{1 / 2}$ of the order parameter $m$. Since $m$ is the unique order 
parameter for electroweak symmetry breaking in the scheme, we may expand the thermodynamical potential $\Omega(T, m) \equiv \Omega(T, \mu=0, m)$ into power-series of $m$ near the critical temperature (phase transition point) as [13]

$$
\Omega(T, m)=\Omega_{0}(T)+a\left(T-T_{c}\right) m^{2}+C(T) m^{4}+\cdots
$$

where $a$ is a positive constant. In Eq. (4.19), no term containing $m$ and $m^{3}$ appears. This is out of the consideration of electroweak symmetry. Since in an effective Higgs field theory of the fermion condensate scheme [9], the order parameter $m$ will be proportional to the vacuum expectation value of the neutral composite Higgs field, the terms in the effective Lagrangian which could lead to $m$ and $m^{3}$ term in Eq. (4.19) have been removed by the $S U_{L}(2) \times U_{Y}(1)$ symmetry. The coefficient of $m^{2}$ term will ensure spontaneous breaking at $T<T_{c}$ and symmetry restoration at $T>T_{c}$. In addition, to make the phase transition point become a stable minimum of $\Omega(T, m)$ at $m=0$, it is necessary that the coefficient $C(T)>0$.

From $\partial \Omega(T, m) / \partial m=0$ we obtain that when $T \leq T_{c}$ the order parameter $m$ can be expressed by

$$
m^{2}=\frac{a}{2 C(T)}\left(T_{c}-T\right)
$$

which will be identical to Eq. (4.18) if we identify

$$
\frac{a}{2 C\left(T_{c}\right)}=b^{2}\left(T_{c}\right) 2 T_{c}
$$

where the $T$ s in $b(T), C(T)$ and $\left(T_{c}+T\right)$ have been replaced approximately by $T_{c}$. This implies that the thermodynamics of the system with order parameter (4.18) can be described by the thermodynamical potential of the form (4.19). Obviously, the entropy given by Eq. (4.19) (to $m^{2}$ order)

$$
S=-[\partial \Omega(T, m) / \partial T]_{V}=\left\{\begin{array}{cc}
S_{0}-2 a T_{c} b^{2}\left(T_{c}\right)\left(T_{c}-T\right) & \text { if } T \leq T_{c} \\
S_{0} & \text { if } T \geq T_{c}
\end{array}\right.
$$

with

$$
S_{0}=-\left[\partial \Omega_{0}(T) / \partial T\right]_{V}
$$

and it will vary continuously when $T$ acrosses $T_{c}$. But the special heat

$$
c_{v}=T(\partial S / \partial T)_{V}=\left\{\begin{array}{cl}
c_{v 0}+2 a T_{c}^{2} b^{2}\left(T_{c}\right) & \text { if } T<T_{c} \\
c_{v 0} & \text { if } T>T_{c}
\end{array}\right.
$$

will experience a jump when $T$ acrosses $T_{c}$. This verifies that the phase transition is a second-order's one indeed. We indicate that the extra factor containing $\ln (\Lambda / T \pi)$ in $b^{2}\left(T_{c}\right)$ does not change the second-order feature of the phase transition and at most, it simply affects the change rate of themodynamical quantities.

\section{Conclusions}

We have shown that a direct analysis of the dynamical fermion mass $m(T, \mu)$ is a quite effective approach for the discussions of electroweak symmetry phase transition. By means of the gap equation at finite temperature, which is proven to be identical in both the imaginary and the real time formalism of thermal field theory, incorporated with the basic relation to define the Higgs vacuum expectation value in the bubble graph approximation of the top-quark condensate scheme, we have given a definite curve of the critical chemical potential $\mu_{c}$ and the critical temperature $T_{c}$ for electroweak symmetry restoration and 
calculated explicit numerical values of $\mu_{c}$ and $T_{c}$. We have also proved by high temperature expansion of the thermal integrals that, for instance, when $\mu=0$ the order parameter $m(T, \mu)$ has the leading $\left(T_{c}^{2}-T^{2}\right)^{1 / 2}$ behavior but with an additional factor dependent on $\ln (\Lambda / T \pi)$ at $T \lesssim T_{c}$. However, despite of the extra factor, the electroweak symmetry restoration at $T \geq T_{c}$ is still a second-order phase transition. This conclusion seems to show the essentiality of the scheme. Certainly, whether it could be changed after the full dynamics of the gauge bosons and the composite Higgs boson are considered may be researched further.

\section{References}

[1] J.C. Taylor, Gauge theories of weak interactions, Cambridge University Press, Cambridge, England (1976); H. Georgi, Weak interactions and modern particle theory, Benjamin/Cummings (1984).

[2] S. Weinberg, Gravitation and Cosmology, Wiley, New York (1972); R.H. Brandenberger, Rev. Mod. Phys. 57 (1985) 1.

[3] D.A. Kirzhnitz and A.D. Linde, Phys. Lett. 42B (1972) 471.

[4] S. Weinberg, Phys. Rev. D9 (1974) 3357.

[5] L. Dolan and R. Jackiw, Phys. Rev. D9 (1974) 3320.

[6] D.A. Kirzhnitz and A.D. Linde, Ann. Phy. 101 (1976) 195; A.D. Linde, Nucl. Phys. B216 (1983) 421 ; Rep. Prog. Phys. 47 (1984) 925.

[7] J.I. Kapusta, Finite-temperature field theory, Cambridge University Press, Cambridge, England (1989) p.170.

[8] Y. Nambu, in New Theory in Physics, Proceedings of XI International Symposium on Elementary Particle Physics, Kazimierz, Poland, 1988, eds Z. Ajduk, S. Pokorski and A. Trautman, World Scientific, Singapore (1989); V.A. Miransky, M. Tanabashi and K. Yamawaki, Mod. Phys. Lett. A4 (1989) 1043; Phys. Lett. B221 (1989) 177.

[9] W.A. Bardeen, C.T. Hill and M. Lindner, Phys. Rev. D41 (1990) 1647.

[10] Y. Nambu and G. Jona-Lasinio, Phys. Rev. 122 (1961) 345; 124 (1961) 246.

[11] N.P. Landsman and van Ch.G. Weert, Phys. Rep. 145 (1987) 141 and references therein.

[12] I.S. Gradshteyn and I.M. Ryzhik, Tables of Integrals, Series and Products, Academic, New york (1980).

[13] L.D. Landau and E.M. Lifshitz, Statistical Physics, Pergamon Press, Oxford (1958). 